For reprint orders, please contact: reprints@futuremedicine.com

\title{
Which way to go in the increasingly complex era of breast cancer heterogeneity
}

Joana Ribeiro*

\section{3th St Gallen International Breast Cancer Conference, St Gallen, Switzerland, 13-16 March 2013}

The St Gallen International Breast Cancer Conference is one of the most important conferences in the breast oncology field, aiming to highlight significant progress achieved in the field and yielding a consensus for the treatment of early breast cancer. This is a biennial conference in which approximately 50 of the best-known specialists in the world (medical oncologists, surgeons, radiation oncologists, pathologists, imaging experts and researchers) vote on the most important topics related to early breast cancer treatment, based on scientific evidence and patient-centered treatment. During the conference, several presentations on the 'state of the art' in breast cancer biology, diagnosis and treatment, as well as poster sessions of selected abstract submissions on related issues, were presented.

In 2011, the International Expert Consensus of St Gallen stated that it was "no longer tenable to consider breast cancer (BC) as a single disease" given the recognition of different biological subtypes within the breast cancer spectrum [1]. Not surprisingly, the 2013 conference was dominated by the remarkable evolution of the molecular and genetic methodologies as ways to refine our classification of the disease and treatment approaches. It's clear that the future of BC treatment is inseparable from the thorough understanding of the molecular mechanisms underpinning carcinogenesis, and that this level of knowledge can only be achieved with a striking 'symbiosis' between the clinical oncology and molecular biology fields. Interestingly, in an era largely dominated by the concept of targeted therapy, the winner of the St Gallen Breast Cancer Award 2013, Aron Goldhirsch, of the European Institute of Oncology, Milan (Italy) gave us a 'Lesson from the past', where he took us through the history of the recognition of the estrogen receptor (ER) and the development of the first targeted therapy in breast cancer, endocrine therapy (ET), highlighting the main challenges that the future holds in this field.

In the traditional starting session, four prominent speakers summarized the 'News since St Gallen 2011'. José Baselga (Memorial Sloan-Kettering Cancer Center, Instituion, NY, USA) and Kent Osborne (Breast Center at Baylor College of Medicine, TX, USA) reviewed the current data related to the PI3K pathway, its role in breast cancer, the frequency with which PI3K is aberrant in breast cancer and the clinical applications of agents that target the PI3K pathway. Both speakers underscored the way PI3KCA mutations not only promote oncogenic transformation by enhancement of downstream signaling elements including Akt, but also play a role in resistance to some of the therapies that block upstream tyrosine kinase receptors - namely antiHER2 or ET. William Foulkes (McGill University, Quebec, Canada) revisited the

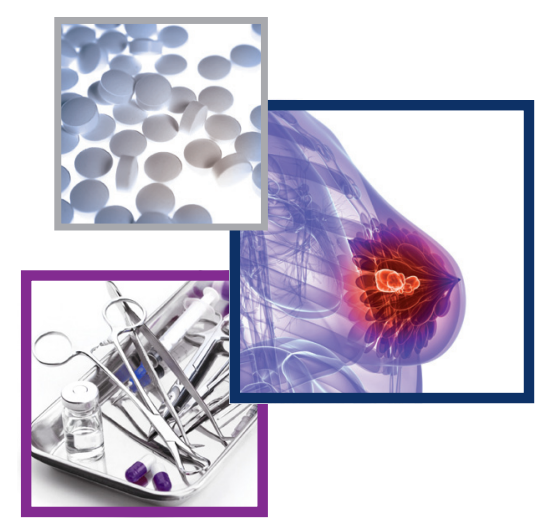

News \& Views

News

Journal Watch

Ask the Experts

Interview

Conference Scene

*Breast Unit, Champalimaud Cancer Center, Lisbon, Portugal; joana.ribeiro@fundacaochampalimaud.pt

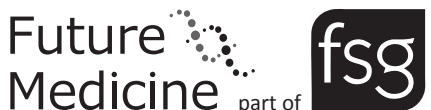


molecular heterogeneity of triple-negative $\mathrm{BC}$ (TNBC) and emphasized the need for further subtyping of this disease in order to better identify molecular-based therapies. He described seven subgroups reported by Lehmann et al. and highlighted the pharmacologically targeted prominent signaling pathways that are derived from the subgroups (e.g., immunotherapy for the immunomodulatory subgroup, therapy directed at the androgen receptor for the luminal androgen receptor subgroup) [2]. Furthermore, deep sequencing of TNBC is undercovering the highly complex genome of these tumors and opening new therapeutic avenues, in particular with PI3K inhibitors that render tumors 'BRCAless'. This innovative and fascinating concept applies in the setting of BRCA-proficient TNBC, in which PI3K inhibition would result in homologous DNA recombination impairment and subsequent sensitization to PARP inhibitors [3]. Finally, Angelo Di Leo (Hospital of Prato, Prato, Italy) provided us with a critical analysis of the clinical utility of the Oxford overview, stressing the need for a practical change in the way we define the risk of relapse, looking not only for the tumor but also for the host.

A full conference day was reserved for the presentation of the latest therapeutic approaches for early BC. Viviana Galimberti (European Institute of Oncology, Milan, Italy) and William Wood (Emory University School of Medicine, GA, USA) discussed surgery, with a focus on how to handle positive sentinel nodes and close/positive margins, while Monica Morrow (Memorial Sloan-Kettering Cancer Center, NY, USA) performed a comprehensive review on how biology is driving the extent of BC surgery. In fact, the successes in targeted therapies and improvements in chemotherapy (CT) have not only improved overall survival, but have also improved local therapy outcomes, and should influence the extent of surgery that is required to manage the disease, as translated from the sentence 'bigger surgery does not overcome bad biology, effective systemic therapy does'.

Adjuvant systemic treatment choices were extensively presented, and much of the discussion centered on the clinical utility of gene-expression-based assays to identify patients with endocrine-responsive $\left(\mathrm{ER}^{+}\right) \mathrm{BC}$ in two different settings: those with sufficiently good prognosis to allow the safe omission of adjuvant CT and those who will probably benefit from extended hormone adjuvant treatment.

For the former, a comprehensive review of the available $\mathrm{BC}$ gene-expression profiling tests (MammaPrint ${ }^{\circledR}$, PAM50 intrinsic subtypes, PAM50 risk of recurrence, OncoypeDx ${ }^{\circledR}$ Recurrence Score, Genomic Grade Index, Breast Cancer Index and EndoPredict ${ }^{\circledR}$ ) was performed. The issues discussed centered on the ability of the various genomic tests to identify patients who may be considered to be most effectively treated with ET alone and the advantages over immunohistochemistry (IHC). Two key points were:

- All current genomic prognostic tests derive their prognostic value from quantifying 'proliferation' and 'ER signaling' and so present a similar prognostic stratification;

- When compared with information provided by IHC-based biomarkers, information coming from multigene-based assays does not just rely on quantifying ER, progesterone receptor (PR), HER2 and $\mathrm{Ki67}$, but rather combines these variables into a statistically multivariate prediction model that may be more objective and reproducible.

However, due to the fact that standardized gene-expression-based tests are not readily available in most of the world as a result of cost, assay turnaround times and other logistic issues, IHC surrogates are still needed and often used. In this regard, a new IHC-based definition of luminal A tumors presented by Charles Perou (University of North Carolina at Chapel Hill, NC, USA) was $\mathrm{ER}^{+}$and/or PR ${ }^{+}$, HER2-, Ki67 < $14 \%$ and PR > 20\% [4].

For the second group of patients, the current question is 'does genomic testing help us to select patients who might benefit from this extended adjuvant approach?'. One limitation that first-generation genomic tests present is that they provide more accurate predictions at 5 years than at 10 years after diagnosis, mainly because they measure proliferation [5,6]. Newer assays, such as the Breast Cancer Index (a combination of genomic grade, molecular grade index and ER-regulated genes) or EndoPredict, appear to better predict the risk of late recurrences [7]. However, we do not yet know if having a higher risk of recurrence necessarily implies that these patients will benefit from extended ET, since sensitivity to ET is also critical to determine benefit.

At the closing consensus session on the 16 March, 51 panelists, predominantly from Europe and the USA, considered a number of questions in order to propose treatment recommendations for patients with early-stage BC. Eric Winer (Dana Farber Cancer Institute, MA, USA) and Aron Goldhirsch served as chairs of the session.

The main panel deliberations with regard to surgery of the primary tumor and axilla, pathology, multigene signatures, ET, CT, anti-HER2 therapy, neoadjuvant systemic therapy, radiotherapy and bisphosphonates are given in the following section.

\section{Surgery of the primary \& axilla}

In 2013, a major change was achieved regarding surgical margin status. The panel was clearly of the view that in women undergoing breast-conserving surgery (BCS), the minimum appropriate surgical margin is no ink on invasive tumor (73\%). Concerning the surgery of the axilla, omission of axillary lymph node dissection in patients with macrometastases in one to two sentinel nodes was only endorsed for patients who underwent radiotherapy after BCS.

\section{Pathology}

The panel strongly supported that distinction between luminal A and B (HER2-) BC can be made by ER, PR and Ki67 expression, although this must only be performed by laboratories participating in quality-of-assurance programs. As in the St Gallen 2011 recommendations, the panel acknowledged the influence of intrinsic subtypes in the decision-making process when adjuvant CT is being considered, although it did not require multigene expression array profiling for its definition 
(clinicopathologic definition was considered sufficient for this purpose). Ki67 was considered useful in the definition of luminal B subtype, although a clear threshold was not possible to establish.

\section{Multigene signatures}

It was considered that the use of multigene signatures should be restricted to $\mathrm{ER}^{+}$, HER2- patients, after a careful clinicopathological assessment. Several of the commercially available first-generation tests can be used.

\section{Endocrine therapy}

For premenopausal patients, more than $80 \%$ of the panel opted for tamoxifen alone as the standard of care, with the great majority (89\%) considering extended treatment duration up to 10 years in some of the patients. The combination of aromatase inhibitors (AI) and ovarian function suppression (OFS) was only regarded as appropriate if patients have a tamoxifen contraindication.

For postmenopausal patients, the majority of the panel considered that tamoxifen is still an option in this setting (93.6\%) and that, in high-risk patients, if an AI is used, it should be up-front (87\%). A little more than half of the panel (58\%) would consider extended AI use beyond 5 years only in the presence of node-positive disease and if given after 5 years of adjuvant tamoxifen or a switching strategy. Extended tamoxifen in this patient population was also endorsed by $51 \%$ of the panelists.

\section{Chemotherapy}

Similar to the 2011 St Gallen statement, the panel agreed that factors arguing for the prescription of CT were high histological grade, high proliferation as measured by $\mathrm{Ki67}$, low hormone receptor status, positive HER2 status and triple-negative status. Both high-risk 21-gene recurrence score and the high-risk 70-gene signature were considered factors indicating the need of CT in an endocrine-responsive population.

Specific recommendations were made based on biologic subgroups of BC, namely:

- Luminal A subgroup was recognized as less responsive to CT (83\%) and considered suited for less intensive regimens, such as doxorubicin and cyclophosphamide (four cycles), cyclophosphamide, methotrexate and 5-fluorouracil (six cycles) or docetaxel and cyclophosphamide (four cycles), if an indication for CT is present;

- The panel agreed that luminal B (HER2-) $\mathrm{BC}$ is, by itself, sufficient to prescribe CT, at least six courses of a sequential anthracycline- and taxane-based regimen should be considered;

- In HER2-amplified BC, the panel could not define a preferred CT regimen and it continued to support the inclusion of both anthracycline (68\%) and taxanes (93\%);

- In basal-like BC, the panel did not endorse platinum-containing chemotherapy, continuing to support the inclusion of anthracyclines and taxanes and an alkylating agent in the standard regimen.

\section{Anti-HER2 therapy}

The panel unanimously supported the use of 1 year of trastuzumab as the standard adjuvant treatment in the HER2 amplified BC patient population. The agreed threshold for anti-HER2 therapy was $5 \mathrm{~mm}(72.5 \%)$, with it being advisable to administer it concurrently with a taxane $(87 \%)$, but not with anthracyclines. In circumstances where chemotherapy could not be delivered, the panel maintained the indication to prescribe trastuzumab.

\section{Neoadjuvant systemic therapy}

Neoadjuvant CT is mainly aimed at facilitating locoregional therapy. If neoadjuvant CT is chosen, all cycles should be given prior to surgery. In the subset of patients with HER2-amplified tumors, an antiHER 2 agent should be incorporated in the neoadjuvant setting. The panel did not support the use of dual HER2 targeting in the preoperative setting.

In postmenopausal patients with highly endocrine-responsive disease, the panel supported neoadjuvant ET alone until the achievement of maximal response.

\section{Radiotherapy}

Radiotherapy after mastectomy continues to be standard in patients with more than four involved lymph nodes and between one and three involved lymph nodes in the presence of adverse pathologic factors $(62 \%)$ or young age $(55 \%)$. The panel agreed that in patients who underwent BCS, short-course radiotherapy (e.g., 40 Gy in 15 fractions) is standard.

\section{Bisphosphonates}

As in 2011, the panel did not support the use of bisphosphonates for their antitumor effect in either pre- or post-menopausal patients.

\section{Financial \& competing interests disclosure}

The author has no relevant affliations or financial involvement with any organization or entity with a financial interest in or financial conflict with the subject matter or materials discussed in the manuscript. This includes employment, consultancies, honoraria, stock ownership or options, expert testimony, grants or patents received or pending, or royalties.

No writing assistance was utilized in the production of this manuscript.

\section{References}

1 Goldhirsch A, Wood WC, Coates AS, Gelber RD, Thurlimann B, Senn HJ. Strategies for subtypes - dealing with the diversity of breast cancer: highlights of the St. Gallen International Expert Consensus on the
Primary Therapy of Early Breast Cancer 2011. Ann. Oncol. 22(8), 1736-1747 (2011).

2 Lehmann BD, Bauer JA, Chen X et al. Identification of human triple-negative breast cancer subtypes and preclinical models for selection of targeted therapies. J. Clin. Invest. 121(7), 2750-2767 (2011).
3 Juvekar A, Burga LN, Hu H et al. Combining a PI3K inhibitor with a PARP inhibitor provides an effective therapy for BRCA1related breast cancer. Cancer Discov. 2(11), 1048-1063 (2012).

4 Prat A, Cheang MC, Martin M et al. Prognostic significance of progesterone receptor-positive 
tumor cells within immunohistochemically defined luminal A breast cancer. J. Clin. Oncol. 31(2), 203-209 (2013).

5 Buyse M, Loi S, van't Veer L et al. Validation and clinical utility of a 70 -gene prognostic signature for women with node-negative breast cancer. J. Natl Cancer Inst. 98(17), 1183-1192 (2006).
6 Desmedt C, Piette F, Loi S et al. Strong time dependence of the 76-gene prognostic signature for node-negative breast cancer patients in the TRANSBIG multicenter independent validation series. Clin. Cancer Res. 13(11), 3207-3214 (2007).
7 Sgroi DC, Sestak I, Cuzick J et al. Comparative performance of Breast Cancer Index (BCI) vs. OncotypeDx and IHC4 in the prediction of late recurrence in hormonal receptor-positive lymph node-negative breast cancer patients: a TransATAC study. Cancer Res. 72(24 Suppl. 3) (2012) (Abstract S1-9). 